\title{
THE PELL EQUATION IN QUADRATIC FIELDS
}

\author{
IVAN NIVEN
}

Consider the equation

$$
\xi^{2}-\gamma \eta^{2}=1
$$

where $\gamma$ is a given integer of a quadratic field $F$, and integral solutions $\xi, \eta$ are sought in $F$. It has been shown ${ }^{1}$ that equation (1) has an infinite number of solutions if and only if $\gamma$ is not totally negative when $F$ is a real field, and $\gamma$ is not the square of an integer of $F$ when $F$ is imaginary. We now obtain the following result:

Let $\gamma$ be such that equation (1) has an infinite number of solutions. If $F$ is a real field it is possible to find a solution $\xi_{1}, \eta_{1}$ of (1) so that every solution is given by the equations

$$
\begin{aligned}
& \xi=\left\{\left(\xi_{1}+\gamma^{1 / 2} \eta_{1}\right)^{n}+\left(\xi_{1}-\gamma^{1 / 2} \eta_{1}\right)^{n}\right\} / 2 \\
& \eta=\left\{\left(\xi_{1}+\gamma^{1 / 2} \eta_{1}\right)^{n}-\left(\xi_{1}-\gamma^{1 / 2} \eta_{1}\right)^{n}\right\} /\left(2 \gamma^{1 / 2}\right),
\end{aligned} \quad n=1,2,3, \cdots,
$$

if and only if $\gamma$ is not a totally positive non-square integer of $F$. If $F$ is imaginary it is always possible to find a solution $\xi_{1}, \eta_{1}$ so that all solutions are given by (2).

The latter result is known to hold for the Pell equation in the rational field. The expression $\gamma^{1 / 2}$ is ambiguous, but no confusion will arise provided it consistently has the same value (we shall specify its value in certain cases). We consider the four sets $\pm \xi, \pm \eta$ to be a single solution, so that equations (2) give "every solution" in the sense that one of the four is present for some value of $n$.

Case 1. F real, $\gamma$ positive but not totally positive. It will be convenient to consider $\gamma^{1 / 2}, \xi$ and $\eta$ positive. We now show that there is but a finite number of solutions of (1) with $\xi$ bounded, say $\xi<N$. For suppose we have an infinitude of solutions $\xi_{i}, \eta_{i}$ with $\xi_{i}<N$ for $i=1,2,3, \cdots$ Taking conjugates in equation (1) we would have

$$
\bar{\xi}_{i}^{2}-\bar{\gamma} \bar{\eta}_{i}^{2}=1,
$$

and since $-\bar{\gamma}$ is positive, this implies that $\bar{\xi}_{i} \leqq 1$ for $i=1,2,3, \cdots$. But it is not possible to have an infinite set of real quadratic integers which, along with their conjugates, are bounded.

Presented to the Society, November 28, 1942; received by the editors August 8, 1942.

${ }^{1}$ Quadratic diophantine equations in the rational and quadratic fields, Trans. Amer. Math. Soc. vol. 52 (1942) p. 2 Theorem 4. We refer to this paper as (Q). 
Hence there exists a least $\xi$, say $\xi_{1}$, among the positive solutions of (1); the corresponding $\eta$, say $\eta_{1}$, is also a least value. Thus we have, among the $\xi+\gamma^{1 / 2} \eta$, a minimum value $\xi_{1}+\gamma^{1 / 2} \eta_{1}$; it is unique, since otherwise $\gamma^{1 / 2}$ would be an element of $F$, contrary to hypothesis. Now any solution $\xi, \eta$ must correspond to a rational integer $n$ such that

$$
\left(\xi_{1}+\gamma^{1 / 2} \eta_{1}\right)^{n} \leqq \xi+\gamma^{1 / 2} \eta<\left(\xi_{1}+\gamma^{1 / 2} \eta_{1}\right)^{n+1} .
$$

Multiplying by $\left(\xi_{1}-\gamma^{1 / 2} \eta_{1}\right)^{n}$ we have

$$
1 \leqq\left(\xi+\gamma^{1 / 2} \eta\right)\left(\xi_{1}-\gamma^{1 / 2} \eta_{1}\right)^{n}<\xi_{1}+\gamma^{1 / 2} \eta_{1} .
$$

The central term is of the form $\xi_{2}+\gamma^{1 / 2} \eta_{2}, \xi_{2}$ and $\eta_{2}$ being integers of $F$. On multiplying this central term by $\xi_{2}-\gamma^{1 / 2} \eta_{2}$ we see that $\xi_{2}, \eta_{2}$ is a solution of (1); also neither $\xi_{2}$ nor $\eta_{2}$ is negative. But since $\xi_{1}+\gamma^{1 / 2} \eta_{1}$ is the least with positive $\xi$ and $\eta$, we must have $\xi_{2}=1$, $\eta_{2}=0$ and hence

$$
\xi+\gamma^{1 / 2} \eta=\left(\xi_{1}+\gamma^{1 / 2} \eta_{1}\right)^{n} .
$$

This implies equations (2).

Case 2. F real, $\gamma$ negative but not totally negative. All solutions of (1) are obtained by taking conjugates of solutions of

$$
\xi^{2}-\bar{\gamma} \eta^{2}=1,
$$

so the problem reduces to Case 1 . The particular solution $\xi_{1}, \eta_{1}$ can be obtained in this case by taking the conjugate of the least positive solution of (3).

Case 3. $F$ real, $\gamma$ a perfect square in $F$ (and hence totally positive). Let $\gamma=\alpha^{2}$, where $\alpha$ is a positive integer in $F$. We prove that there is only a finite number of positive solutions of (1) with $\xi+\alpha \eta$ bounded. Any solution gives us two integers of the field $\xi+\alpha \eta$ and $\xi-\alpha \eta$, with product unity. But two integers of a real quadratic field have this property only if one is the conjugate, or the negative of the conjugate, of the other. Hence we can write $\xi+\alpha \eta=\rho, \xi-\alpha \eta= \pm \bar{\rho}$. Taking $\xi, \eta$ and $\alpha$ positive we have $\rho>1$ and $1>|\bar{\rho}|>0$. But there is only a finite number of integers $\rho$ which exceed 1 , are bounded, and possess the property $\rho \bar{\rho}= \pm 1$. Thus among the infinitude of solutions of (1) there exists one having $\xi+\alpha \eta$ a minimum. It is unique; for if $\xi_{1}+\alpha \eta_{1}$ $=\xi_{2}+\alpha \eta_{2}$ with, say, $\xi_{1}>\xi_{2}$ and $\eta_{1}<\eta_{2}$, then not both $\xi_{1}, \eta_{1}$ and $\xi_{2}, \eta_{2}$ can satisfy (1). Hence we proceed as in Case 1 to derive all solutions from this unique smallest one.

Case 4. F real, $\gamma$ totally positive but not a perfect square in $F$. We shall show that (1) has infinitely many solutions with $\xi$ bounded. In Lemma 2 in $\$ 3$ of $(Q)$ it is proved that if $\gamma$ is positive but not 
totally positive there are infinitely many solutions of (1); we shall make direct use of this proof.

Note first that the infinitude of solutions obtained are such that $\bar{\xi}$ is bounded. To prove this, we observe that the integer $\rho$ introduced in connection with the infinite series (10) in $(Q)$ is bounded, as also is its conjugate; this is seen from inequality (9) and the argument following. And since $\xi$ is obtained in (15) by dividing $\xi_{r} \xi_{s}-\gamma \eta_{r} \eta_{s}$ by $\rho$ we can obtain $\bar{\xi}$ by multiplying $\bar{\xi}_{r} \bar{\xi}_{s}-\bar{\gamma} \bar{\eta}_{r} \bar{\eta}_{s}$ by the reciprocal of $\bar{\rho}$, both of which are bounded: the first because of (8), and the second because the reciprocal of $\bar{\rho}$ does not exceed $\rho$, which is bounded. Instead of obtaining the infinitude of solutions as suggested in $(Q)$ (see the sentence immediately preceding Lemma 3), we can get them by compounding the first pair in (11) with all subsequent pairs, and thus $\bar{\xi}$ is bounded for all resulting solutions.

Next we note that the proof of Lemma 2 in $(Q)$ is valid if $\gamma$ is a totally positive non-square integer of $F$. Two remarks should be made in explanation: after inequality (9) the argument used to show that the left side of (7) cannot be zero needs a slight alteration; the result follows from the fact that $\gamma^{1 / 2}$, or $\delta$, cannot be an element of $F$, by hypothesis; also the inequality just preceding (10) must be changed since $\bar{\gamma}$ is now positive; the essential idea, that $\bar{\xi}^{2}-\bar{\gamma} \bar{\eta}^{2}$ is bounded is still correct.

Thus the proof of Lemma 2 of $(Q)$ can be used to obtain the result that our present equation (1) has infinitely many solutions with $\bar{\xi}$ bounded, $\gamma$ being totally positive but not a square. But $\bar{\xi}$ and $\bar{\eta}$ are solutions of

$$
\bar{\xi}^{2}-\bar{\gamma} \bar{\eta}^{2}=1,
$$

$\bar{\gamma}$ also being totally positive but not a square; interchanging $\xi, \eta$ and $\gamma$ with their conjugates we have the result that our equation (1) has infinitely many solutions with $\xi$ bounded.

Now if there were some least positive solution $\xi_{1}, \eta_{1}$, we could proceed as in Case 1 and derive all solutions from it by equations (2). But $\xi$ in equations (2) is bounded for only a finite number of values of $n$. Thus we cannot obtain all solutions by such a scheme.

Case 5. F imaginary, $\gamma$ not a perfect square in $F$. We first show that, except for trivial solutions $\pm 1,0$, we cannot have $\left|\xi+\gamma^{1 / 2} \eta\right|=1$ : For otherwise $\left|\xi-\gamma^{1 / 2} \eta\right|=1$, and the inequality

$$
|\xi| \leqq(1 / 2)\left|\xi+\gamma^{1 / 2} \eta\right|+(1 / 2)\left|\xi-\gamma^{1 / 2} \eta\right|
$$

would give us $|\xi| \leqq 1$, yielding only a trivial solution. Hence for convenience we can choose the sign of $\xi$ (or $\eta$ ) so that 


$$
\left|\xi+\gamma^{1 / 2} \eta\right|>1, \quad\left|\xi-\gamma^{1 / 2} \eta\right|<1 .
$$

Next we show that two essentially different solutions $\xi_{1}, \eta_{1}$ and $\xi_{2}, \eta_{2}$ cannot be such that

$$
\left|\xi_{1}+\gamma^{1 / 2} \eta_{1}\right|=\left|\xi_{2}+\gamma^{1 / 2} \eta_{2}\right| \text {. }
$$

For if this were the case we would have another solution $\xi_{3}, \eta_{3}$ defined by

$$
\xi_{3}=\xi_{1} \xi_{2}-\gamma \eta_{1} \eta_{2}, \quad \eta_{3}=\xi_{1} \eta_{2}-\xi_{2} \eta_{1} .
$$

It would follow that

$$
\left|\xi_{3}+\gamma^{1 / 2} \eta_{3}\right|=\left|\xi_{1}-\gamma^{1 / 2} \eta_{1}\right| \cdot\left|\xi_{2}+\gamma^{1 / 2} \eta_{2}\right|=1,
$$

and by the argument above, $\xi_{3}= \pm 1$ and $\eta_{3}=0$. These imply that $\xi_{1}= \pm \xi_{2}, \eta_{1}= \pm \eta_{2}$.

Now $\left|\xi+\gamma^{1 / 2} \eta\right|$ is less than any given positive value $N>1$ for but a finite number of solutions of (1); for by (4) if $\left|\xi+\gamma^{1 / 2} \eta\right|$ is bounded so is $|\xi|$, and this cannot be bounded for an infinite set of integers of an imaginary quadratic field. Hence there exists a unique nontrivial solution of (1) having $\left|\xi+\gamma^{1 / 2} \eta\right|$ a minimum, and we can proceed as in Case 1.

Purdue University 